\title{
Pacific
}

Journal of

Mathematics

\section{REFLEXIVE OPERATOR ALGEBRAS ON BANACH SPACES}

Florence Merlevède, Costel Peligrad and Magda Peligrad 


\title{
REFLEXIVE OPERATOR ALGEBRAS ON BANACH SPACES
}

\author{
Florence Merlevède, Costel Peligrad and Magda Peligrad
}

\begin{abstract}
In this paper we study the reflexivity of a unital strongly closed algebra of operators with complemented invariant subspace lattice on a Banach space. We prove that if such an algebra contains a complete Boolean algebra of projections of finite uniform multiplicity and with the direct sum property, then it is reflexive, i.e., it contains every operator that leaves invariant every closed subspace in the invariant subspace lattice of the algebra. In particular, such algebras coincide with their bicommutant.
\end{abstract}

\section{Introduction}

Let $A \subset B(X)$ denote a strongly closed algebra of operators on the Banach space X. Suppose that $A$ has the property that each of its invariant subspaces has an invariant complement. If $A$ contains a complete Boolean algebra of projections of finite uniform multiplicity and with the direct sum property as defined below, we prove that $A$ is reflexive in the sense that it contains all the operators which leave its closed invariant subspaces invariant (Theorem 15). In particular such an algebra is equal to its bicommutant $A^{\prime \prime}$ (Corollary 22). The problem of whether a strongly closed algebra of operators with complemented invariant subspace lattice is reflexive started to be studied in the sixties. This problem is a generalization of the invariant subspace problem in operator theory. Arveson [1967] introduced a technique for studying the particular case of transitive algebras on Hilbert spaces, namely the strongly closed algebras of operators on Hilbert spaces that have no nontrivial closed invariant subspaces. He proved that every transitive algebra that contains a maximal abelian von Neumann algebra coincides with the full algebra $B(X)$ if $X$ is a complex Hilbert space. Douglas and Pearcy [1972] extended the result of Arveson to the case of transitive operator algebras containing an abelian von Neumann algebra of finite multiplicity. Hoover [1973] extended the result of Douglas and Pearcy to the case of reductive operator algebras on Hilbert spaces that contain

C. Peligrad and M. Peligrad were supported in part by a Charles Phelps Taft Memorial Fund grant. M. Peligrad was also supported by NSF grant DMS-1208237.

MSC2010: primary 47A15, 47B48; secondary 47C05.

Keywords: operator algebras, invariant subspace lattice, Boolean algebra of projections, spectral operator. 
abelian von Neumann algebras of finite multiplicity. Hoover proved that every reductive operator algebra (that is a strongly closed subalgebra for which every closed invariant subspace is reducing) which contains an abelian von Neumann algebra of finite multiplicity is self-adjoint. The transitive algebra result of Douglas and Pearcy was generalized in [Önder and Orhon 1989] to the case of transitive algebras on Banach spaces that contain a $n$-fold direct sum of a cyclic complete Boolean algebra of projections. The case of operator algebras on Banach spaces with complemented invariant subspace lattice was considered by Rosenthal and Sourour [1977]. They proved that every strongly closed algebra of operators with complemented invariant subspace lattice containing a complete Boolean algebra of projections of uniform multiplicity one is reflexive.

In this paper we build upon the techniques introduced by Arveson and developed in [Douglas and Pearcy 1972; Radjavi and Rosenthal 1973] for invariant subspaces of operator algebras as well as Bade's multiplicity theory of Boolean algebras of projections [Bade 1955; 1959]. We also use the results of [Foguel 1959] and [Tzafriri 1967] about the commutant of Boolean algebras of projections of finite multiplicity.

\section{Notation and preliminary results}

2.1. Invariant subspaces of operator algebras. Let $X$ be a complex Banach space and $B(X)$ the algebra of all bounded linear operators on $X$. We will denote by $X^{(n)}$ the direct sum of $n$ copies of $X$ and, if $S \subset B(X)$, we set

$$
S^{(n)}=\left\{a \oplus a \oplus \cdots \oplus a \in B\left(X^{(n)}\right) \mid a \in S\right\} .
$$

If $S \subset B(Y)$, where $Y$ is a Banach space, we denote by Lat $S$ the collection of all closed linear subspaces of $Y$ that are invariant under every element of $S$. If $L$ is a collection of closed linear subspaces of $Y$, we denote by $\operatorname{alg} L$ the (strongly closed) algebra of operators on $Y$ that leave every element of $L$ invariant. An algebra $A \subset B(X)$ is called reflexive if algLat $A=A$.

In what follows all the subalgebras $A \subset B(X)$ will be assumed to be strongly closed and containing the identity operator $I \in B(X)$.

Remark 1. Let $A \subset B(X)$ be a strongly closed algebra with $I \in A$ and $b \in B(X)$. If Lat $A^{(n)} \subset$ Lat $b^{(n)}$ for every $n \in \mathbb{N}$, then $b \in A$.

Proof. Indeed, then for every finite set of elements $\left\{x_{1}, x_{2}, \ldots, x_{n}\right\} \subset X$ we have that $K=\overline{\left\{a x_{1} \oplus a x_{2} \oplus \cdots \oplus a x_{n} \mid a \in A\right\}} \in$ Lat $A^{(n)}$ and therefore $K \in$ Lat $b^{(n)}$. This means that $b \in A$, since $A$ is strongly closed.

Proposition 2. Let $A \subset B(X)$ be a strongly closed algebra with complemented invariant subspace lattice and with $I \in A$. Let $q \in B(X)$ be a projection. 
(i) If $q \in A$, the algebra $q A q \subset B(q X)$ has complemented invariant subspace lattice and $\operatorname{algLat}(q A q)=q(\operatorname{algLat} A) q$.

(ii) If $q \in A^{\prime}$, where $A^{\prime}$ denotes the commutant of $A$, the strong operator closure $\overline{q A q}^{\text {so }} \subset B(q X)$ is an algebra with complemented invariant subspace lattice.

Proof. We prove first (i). Clearly, $q A q$ is a strongly closed subalgebra of $B(q X)$ whose unit is $q$. Let $L \subset q X, L \in \operatorname{Lat}(q A q)$. We define $\tilde{L}=\overline{A q L}$, the closure being taken in $X$. Then, obviously, $\tilde{L} \in$ Lat $A$ and, therefore $\tilde{L}$ has a complement $\tilde{L}^{c}$ in Lat $A$. Since $q \in A$, we have $q \tilde{L} \subset \tilde{L}$, also $q \tilde{L}^{c} \subset \tilde{L}^{c}$ and $q \tilde{L}^{c} \in \operatorname{Lat}(q A q)$. Moreover, it is immediate that $q \tilde{L}$ and $q \tilde{L}^{c}$ are closed linear subspaces of $q X$ such that

$$
q \tilde{L} \oplus q \tilde{L}^{c}=q X .
$$

On the other hand we have $L \subset q \tilde{L}=q \overline{A q L} \subset \overline{q A q L} \subset \bar{L}=L$. Hence

$$
q \tilde{L}=L \text {. }
$$

It follows that $L$ is complemented in $\operatorname{Lat}(q A q)$ and so $q A q$ has complemented invariant subspace lattice. Let now $b \in \operatorname{algLat} A$ and $L \in \operatorname{Lat}(q A q)$. By the above argument, there exists $\widetilde{L} \in \operatorname{Lat} A$ such that $L=q \tilde{L}$. Hence $b \widetilde{L} \subset \tilde{L}$. Therefore, since $q \tilde{L}=L \subset \tilde{L}$ it follows that $q b q L \subset L$, so $q b q \in \operatorname{algLat}(q A q)$. Conversely, let $c \in \operatorname{algLat}(q A q)$ and let $\tilde{c} \in B(X)$ be the extension of $c$ to $X$ that equals 0 on $(I-q) X$. Then, it is straightforward to show that $\tilde{c} \in \operatorname{algLat} A$ and $c=q \tilde{c} q$ and so the proof is completed.

To establish (ii), let $K \in \operatorname{Lat}(q A q)$. Since $q \in A^{\prime}$, it follows that $K \in$ Lat $A$ and therefore $K$ has a complement $K^{c} \in$ Lat $A$. Then, clearly $K^{c} \cap q X \in \operatorname{Lat}(q A q)$ and $K+K^{c} \cap q X=q X$.

We will also need the following:

Lemma 3. Let $A \subset B(X)$ be an algebra with complemented invariant subspace lattice and let $K \in$ Lat $A$. If $p \in A^{\prime}$ is the projection on $K$ and $t_{1}, t_{2}, \ldots, t_{n} \in(p A p)^{\prime}$, for some $n \in \mathbb{N}$, then the subspace

$$
\Gamma_{\left\{t_{1}, t_{2}, \ldots, t_{n} ; p\right\}}=\left\{x \oplus t_{1} x \oplus t_{2} x \oplus \cdots \oplus t_{n} x \mid x \in p X\right\} \in \text { Lat } A^{(n+1)}
$$

is complemented in Lat $A^{(n+1)}$.

Proof. Since $A$ has complemented invariant subspace lattice and $p X=K \in$ Lat $A$, it follows that the subspace $(1-p) X=(p X)^{c}=K^{c}$ belongs to Lat $A$. It is then clear that $(p X)^{c} \oplus X^{(n)}$ is a complement of $\Gamma_{\left\{t_{1}, t_{2}, \ldots, t_{n} ; p\right\}}$ in Lat $A^{(n+1)}$.

Remark 4. Let $I \in A \subset B(X)$ be a strongly closed subalgebra with complemented invariant subspace lattice. If $A$ is reflexive, then $A^{\prime \prime}=A$ where $A^{\prime \prime}$ denotes the bicommutant of $A$. 
Proof. If $a \in A^{\prime \prime}$, then, in particular, $a$ commutes with every projection on an invariant subspace of $A$. Therefore $a \in \operatorname{algLat} A=A$.

The following concept is defined in [Radjavi and Rosenthal 1973, §8.2], for instance.

Definition 5. Let $A \subset B(X)$ be a subalgebra. A linear operator $T$ defined on a not necessarily closed linear subspace $P \subset X$ is called a graph transformation for $A$ if there exist finitely many linear operators $T_{1}, T_{2}, \ldots, T_{l}$, all defined on $P$, such that

$$
\left\{x \oplus T x \oplus T_{1} x \oplus T_{2} x \oplus \cdots \oplus T_{l} x \mid x \in P\right\} \in \text { Lat } A^{(l+2)} .
$$

Remark 6. Let $K \in$ Lat $A^{(n)}, n \in \mathbb{N}$. Define

$$
K_{0}=\left\{\boldsymbol{x} \in X^{(n-1)} \mid 0 \oplus \boldsymbol{x} \in K\right\} \in \text { Lat } A^{(n-1)} .
$$

Then, if $K_{0}$ is complemented in Lat $A^{(n-1)}$ with complement $K_{0}^{c}$ it follows that there exist graph transformations for $A: T_{1}, T_{2}, \ldots, T_{n-1}$, defined on a linear subspace, $P \subset X$, such that

$$
\left(X \oplus K_{0}^{c}\right) \cap K=\left\{x \oplus T_{1} x \oplus T_{2} x \oplus \cdots \oplus T_{n-1} x \mid x \in P\right\} .
$$

Proof. Straightforward.

\subsection{Boolean algebras of projections in Banach spaces and spectral operators.} Let $\mathscr{B}$ be a complete Boolean algebra of projections in a (complex) Banach space $X$ (as defined for instance in [Bade 1955] or in [Dunford and Schwartz 1988, Chapter XVII]). It is known [Stone 1949] that there exists an extremally disconnected compact Hausdorff topological space $\Omega$ (that is a compact Hausdorff space in which the closure of every open set in it is open), such that $\mathscr{B}$ is equivalent as a Boolean algebra with the Boolean algebra of open and closed subsets of $\Omega$. We will denote by $\Sigma$ the collection of Borel sets of $\Omega$. Such a compact Hausdorff space is called a Stonean space.

The following remark collects some results about the complete Boolean algebras of projections in Banach spaces that will be used in this paper.

Remark 7. (i) If $\mathscr{B}$ is a complete Boolean algebra of projections, then there is a regular countably additive spectral measure $E$ in $X$ defined on the family of Borel sets in $\Omega$ such that the mapping

$$
S(f)=\int_{\Omega} f(w) E(d w)
$$

is a continuous isomorphism of the algebra $C(\Omega)$ of continuous functions on $\Omega$ onto the uniformly closed algebra of operators, $B$, generated by $\mathscr{B}$.

(ii) The algebra $B$ coincides with the strongly closed algebra generated by $\mathscr{B}$ and consists of spectral operators of scalar type. 
(iii) The range of $E$ is precisely the Boolean algebra $\mathscr{B}$.

(iv) $\mathscr{B}$ is norm bounded.

Proof. (i) and (iii) follow from [Dunford and Schwartz 1988, Lemma XVII.3.9]. Point (ii) is Corollary XVII.3.17 of the same reference, and (iv) follows from [Bade 1955, Theorem 2.2].

Let $\mathscr{B} \subset B(X)$ be a complete Boolean algebra of projections that contains the identity projection $I \in B(X)$. We say that $I \in \mathscr{B}$ has multiplicity $k, k \in \mathbb{N}$, if there are $x_{1}, x_{2}, \ldots, x_{k} \in X$ such that $\overline{\operatorname{lin}}\left\{e x_{i} \mid e \in \mathscr{B}, 1 \leq i \leq k\right\}=X$ and no subset of $X$ of cardinality less than $k$ has this property [Bade 1959, Definition 3.2]. The Boolean algebra $\mathscr{B}$ is said to be of uniform multiplicity $k$ if every projection $e \in \mathscr{B}, e \neq 0$ has multiplicity $k$. For each $i, 1 \leq i \leq k$, define $\mathfrak{M}\left(x_{i}\right)=\varlimsup \operatorname{lin}\left\{e x_{i} \mid e \in \mathscr{B}\right\}$. Here, $\varlimsup\left\{e x_{i} \mid e \in \mathscr{B}\right\}$ denotes the closed linear subspace of $X$ spanned by $\left\{e x_{i} \mid e \in \mathscr{B}\right\}$.

The next remark collects some known results from [Bade 1959] (see also [Dunford and Schwartz 1988]).

Remark 8. Let $\mathscr{B}$ be a complete Boolean algebra of finite uniform multiplicity $n$, $n \in \mathbb{N}$, and let $\left\{x_{1}, \ldots, x_{n}\right\}$ be a set of vectors such that

$$
\varlimsup\left\{e x_{i} \mid e \in \mathscr{B}, 1 \leq i \leq n\right\}=X .
$$

(i) There are $x_{i}^{*} \in X^{*}, i=1,2, \ldots, n$, where $X^{*}$ is the dual Banach space of $X$, such that each of the measures $\mu_{i}(\delta)=x_{i}^{*} E(\delta) x_{i}, i \in\{1,2, \ldots, n\}, \delta \in \Sigma$ vanishes on sets of first category of $\Omega$ and $\mu_{i}(\sigma) \neq 0$ if $\sigma$ has nonempty interior. The measures $\mu_{i}$ are equivalent and $x_{i}^{*}\left(\mathfrak{M}\left(x_{j}\right)\right)=\{0\}$ for $i \neq j$.

(ii) There exists a continuous injective linear map $V$ of $X$ onto a dense linear subspace $L \subset \sum_{i=1}^{n} L^{1}\left(\Omega, \Sigma, \mu_{i}\right)$ such that if $V(x)=f=\sum f_{i}$, then:

$$
x_{i}^{*} E(\delta) x=\int_{\delta} f_{i}(\omega) \mu_{i}(d \omega),
$$

for $\delta \in \Sigma$. In particular, $V\left(x_{i}\right)=0 \oplus \cdots \oplus \chi_{\Omega} \oplus \cdots \oplus 0$, where $\chi_{\Omega}=1$ is in the $i$-th place in the direct sum.

$$
x=\lim _{m \rightarrow \infty} \sum_{i=1}^{n} S\left(f_{i} \chi_{\delta_{m}}\right) x_{i},
$$

where $\chi_{\delta_{m}}$ is the characteristic function of

$$
\delta_{m}=\left\{\omega|| f_{i}(\omega) \mid \leq m, i=1,2, \ldots, n\right\} .
$$

(iii) The linear space $L$ is a Banach space when endowed with the norm

$$
\|\boldsymbol{f}\|_{0}=\max _{1 \leq i \leq n}\left\|f_{i}\right\|_{1}+\left\|V^{-1}(\boldsymbol{f})\right\|,
$$

and $V$ is a Banach space isomorphism between $X$ and $\left(L,\|\cdot\|_{0}\right)$. 
Proof. Points (i) and (ii) follow from [Bade 1959, Lemma 5.1 and Theorem 5.2] (see also [Dunford and Schwartz 1988, Theorem XVIII.3.19]). The proof of (iii) is immediate.

A function $f$ is called $E$-essentially bounded if

$$
\inf _{E(\delta)=1} \sup _{\omega \in \delta}|f(\omega)|
$$

is finite [Dunford and Schwartz 1988, Definition 7].

Denote by $E B(\Omega, \Sigma)$ the set of all $E$-essentially bounded $\Sigma$-measurable functions.

Lemma 9. With the notations in Remark 8 , if $\varphi \in E B(\Omega, \Sigma)$, then the operator $M_{\varphi}(\boldsymbol{f})=\varphi \boldsymbol{f}$ is a well defined, bounded operator on $\left(L,\|\cdot\|_{0}\right)$ and $M_{\varphi}=$ $V S(\varphi) V^{-1}$. Here $\varphi \boldsymbol{f}=\varphi f_{1} \oplus \varphi f_{2} \oplus \cdots \oplus \varphi f_{n}$. Thus

$$
V B V^{-1}=\left\{M_{\varphi} \mid \varphi \in E B(\Omega, \Sigma)\right\} .
$$

Proof. Let $\boldsymbol{f} \in L$ and $x=S(\varphi) V^{-1}(\boldsymbol{f})$. Then, according to point (a) in Remark 8 (ii), if $\boldsymbol{g}=V(x)$, we have $x_{i}^{*} S\left(\chi_{\delta}\right) x=\int_{\delta} g_{i}(w) \mu_{i}(d w)$ for every Borel set $\delta \in \Sigma$. On the other hand,

$$
x_{i}^{*} S\left(\chi_{\delta}\right) x=x_{i}^{*} S\left(\chi_{\delta}\right) S(\varphi) V^{-1}(\boldsymbol{f})=x_{i}^{*} S\left(\chi_{\delta} \varphi\right) V^{-1}(\boldsymbol{f})=\int_{\delta} \varphi(w) f_{i}(w) \mu_{i}(d w) .
$$

Hence $g_{i}=\varphi f_{i} \mu_{i}$-a.e., so $\boldsymbol{g}=\varphi \boldsymbol{f}$ a.e. and the proof is completed.

In [Dieudonné 1956] is presented an example of a Boolean algebra of projections, $\mathscr{B}$, such that every nonzero projection $e \in \mathscr{B}$ has multiplicity 2 . However, for no choice of $x_{1}, x_{2} \in X$ or $e \in \mathscr{B}, e \neq 0$ is $e X$ the algebraic sum of $\mathfrak{M}\left(e x_{1}\right)$ and $\mathfrak{M}\left(e x_{2}\right)$. In the rest of this paper we will consider only Boolean algebras of finite uniform multiplicity with the direct sum property:

Definition 10. We say that the complete Boolean algebra $\mathscr{B}$ of uniform multiplicity $k$ has the direct sum property if $X$ is the algebraic (and therefore, Banach) direct sum of $\mathfrak{M}\left(x_{i}\right), 1 \leq i \leq k$.

A particular case of a Boolean algebra of uniform multiplicity $k$ with the direct sum property is the $k$-fold direct sum of $k$ copies of a cyclic Boolean algebra of projections. Other examples are presented in [Foguel 1959].

Lemma 11. Suppose that $\mathscr{B}$ is a complete Boolean algebra of projections of uniform multiplicity $k$ with the direct sum property. Then, for every $\epsilon>0$ there exist $e \in \mathscr{B}$, $e=E(\rho)$, and $\rho \in \Sigma$ with $\mu_{l}\left(\rho^{c}\right)<\epsilon$ for every $1 \leq l \leq k$ (where $\rho^{c}$ is the complement of $\rho)$ such that for every $\left\{\varphi_{i j} \mid 1 \leq i, j \leq k\right\} \subset E B(\Omega, \Sigma)$, the matrix $\left[\varphi_{i j} \chi_{\rho}\right]$ is a bounded linear operator on $\left(L,\|\cdot\|_{0}\right)$ and $\left[\varphi_{i j} \chi_{\rho}\right]$ belongs to the commutant $\mathscr{B}^{\prime}$ of $\Re$. 
Proof. Since the measures $\mu_{l}, 1 \leq l \leq k$ are equivalent, let $h_{m l}=d \mu_{m} / d \mu_{l}$, $1 \leq m, l \leq k$ be the corresponding Radon Nikodym derivative. Let $\epsilon>0$ be arbitrary. Fix $1 \leq m, l \leq k$. Then, since $\bigcup_{n=1}^{\infty}\left\{1 / n \leq h_{m l} \leq n\right\}=\Omega$, there is a $n \in \mathbb{N}$ such that $\mu_{l}\left(\left\{1 / n \leq h_{m l} \leq n\right\}^{c}\right)<\epsilon / k^{2}$. Therefore there is a $n \in \mathbb{N}$ such that $\mu_{l}\left(\left\{1 / n \leq h_{m l} \leq n\right\}^{c}\right)<\epsilon$ for every $1 \leq m, l \leq k$. Let $\rho=\left\{1 / n \leq h_{m l} \leq n\right\} \in \Sigma$. It is easy to see that for every Borel subset $\sigma \subset \rho$ we have $\mu_{i}(\sigma) / n \leq \mu_{j}(\sigma) \leq n \mu_{i}(\sigma)$ for all $1 \leq i, j \leq k$. Hence all the spaces $M_{\chi_{\rho}} L^{1}\left(\mu_{i}\right)=\chi_{\rho} L^{1}\left(\mu_{i}\right), 1 \leq i \leq k$, are equal as sets and mutually isomorphic as Banach spaces. Then, clearly,

$$
\chi_{\rho} L=\chi_{\rho} L^{1}\left(\mu_{1}\right) \oplus \chi_{\rho} L^{1}\left(\mu_{2}\right) \oplus \cdots \oplus \chi_{\rho} L^{1}\left(\mu_{k}\right) .
$$

Since $\mathscr{B}$ has the direct sum property, we also have

$$
E(\rho) X=E(\rho) \mathfrak{M}\left(x_{1}\right) \oplus \cdots \oplus E(\rho) \mathfrak{M}\left(x_{k}\right)
$$

and the lemma follows.

For the definition and basic facts about spectral operators on Banach spaces we refer to [Dunford and Schwartz 1988]. We will need the following result, which follows from [Tzafriri 1967, Theorem 2] and [Foguel 1959, Lemma 2.1 and Theorem 2.3].

Remark 12. Let $T \in B(X)$ and let $\mathscr{B}$ be a complete Boolean algebra of projections in $X$, of uniform multiplicity $k, k \in \mathbb{N}$. If $T$ commutes with the strongly closed algebra $B$ generated by $\mathscr{B}$, then there exists an increasing sequence of projections $\left\{e_{m}=E\left(\chi_{\delta_{m}}\right) \mid m \in \mathbb{N}\right\} \subset \mathscr{B}$ such that $\left\{e_{m}\right\}$ converges strongly to the identity $I \in B(X)$ and $T e_{m}$ is a spectral operator of finite type for every $m$. Moreover, if $T \in B^{\prime}$ is a spectral operator then $T$ is the sum of a spectral operator $R$ of scalar type in $B^{\prime}$ and a nilpotent operator $Q$ of order $k, Q \in B^{\prime}$.

Next we will study the dense linear subspaces of $X$ that are invariant under every element of $B$, where $B$ is the strongly closed algebra generated by $\mathscr{B}$, the complete Boolean algebra of projections of uniform multiplicity $k$ with the direct sum property. The following lemma is an extension to the case of Banach spaces and an improvement on [Douglas and Pearcy 1972, Lemma 3.3]. Using Remark 8 and Lemma 9, we will identify $X$ with $L$ and $B$ with $\left\{V S(\varphi) V^{-1} \mid S(\varphi) \in B\right\}$.

Lemma 13. Let $k \in \mathbb{N}$ and $B$ the strongly closed algebra generated by the Boolean algebra of projections of uniform multiplicity $k, \mathscr{B} \subset B(X)$ and with the direct sum property. With the above notations, suppose that $\mathscr{D} \subset X$ is a dense linear subspace which is invariant under all operators in $B$. Then, for every $\epsilon>0$, there exists an open and closed set $\lambda_{\epsilon} \subset \Omega$ such that

(i) $\mu_{i}\left(\lambda_{\epsilon}^{c}\right)<\epsilon, i=1,2, \ldots, k$, where $\lambda_{\epsilon}^{c}$ is the complement of $\lambda_{\epsilon}$ in $\Omega$, and 
(ii) $\chi_{\lambda_{\epsilon}} \boldsymbol{e}_{j} \in \mathscr{D}$ for all $j \in\{1,2, \ldots, k\}$, where $\left\{\boldsymbol{e}_{j} \mid j=1,2, \ldots, k\right\}$ is the standard basis of $\mathbb{C}^{(k)}$.

Proof. If $z=\left(z^{1}, z^{2}, \ldots, z^{k}\right) \in \mathbb{C}^{(k)}$, consider the norm

$$
\|z\|=\max \left\{\left|z^{p}\right| \mid 1 \leq p \leq k\right\} .
$$

It is easy to see that there exists $\alpha>0$ such that if the set $\left\{h_{1}, h_{2}, \ldots, h_{k}\right\} \subset \mathbb{C}^{(k)}$ satisfies $\left\|h_{i}-\boldsymbol{e}_{i}\right\|<\alpha, i=1,2, \ldots, k$, then the set $\left\{h_{1}, h_{2}, \ldots, h_{k}\right\}$ is linearly independent. Let now $\epsilon>0$ be arbitrary. We can choose $\alpha<\epsilon^{2} / 2$. Let $\rho \in \Sigma$, $\mu_{l}(\rho)<\epsilon / 2,1 \leq l \leq k$, be as in Lemma 11. Since $\Omega$ is extremally disconnected, we can assume that $\rho$ is an open and closed set. For every $j, 1 \leq j \leq k$ let $\boldsymbol{g}_{j}(w)=\boldsymbol{e}_{j}$, if $w \in \rho$ and $\boldsymbol{g}_{j}(w)=0$ if $\omega \in \rho^{c}$. Since by point (a) of Remark 8 (ii) we have that $\boldsymbol{g}_{j} \in \chi_{\rho} L$ for every $j, 1 \leq j \leq k$ and $\chi_{\rho} \mathscr{D}$ is dense in $\chi_{\rho} L$, it follows that there exists a set of elements $\left\{\boldsymbol{l}_{i} \mid 1 \leq i \leq k\right\} \subset \chi_{\rho} \mathscr{D}, \boldsymbol{l}_{i}=l_{i}^{1} \oplus l_{i}^{2} \oplus \cdots \oplus l_{i}^{k}$ such that

$$
\left\|\boldsymbol{l}_{i}-\boldsymbol{g}_{i}\right\|_{0}=\max _{1 \leq p \leq k}\left\{\left\|l_{i}^{p}-g_{i}^{p}\right\|_{0}=\left\|l_{i}^{p}-g_{i}^{p}\right\|_{1}+\left\|T^{-1}\left(l_{i}^{p}-g_{i}\right)^{p}\right\|\right\}<\alpha<\epsilon^{2} .
$$

Let $\delta_{\epsilon}=\bigcap_{i=1}^{k}\left\{\omega \in \rho|| l_{i}^{p}(w)-g_{i}^{p}(w) \mid \geq \epsilon\right.$ and $\left.1 \leq p \leq k\right\}$. Then we have

$$
\epsilon^{2} / 2>\alpha>\max \left\{\left\|l_{i}^{p}-g_{i}^{p}\right\|_{1} \mid 1 \leq i, p \leq k\right\} \geq \epsilon \mu_{m}\left(\delta_{\epsilon}\right) \text { for } 1 \leq m \leq k .
$$

Hence $\mu_{m}\left(\delta_{\epsilon}\right)<\epsilon / 2$ for $m=1,2, \ldots, k$. Assuming that $\epsilon<2$, it follows that $\mu_{m}\left(\delta_{\epsilon}^{c}\right) \neq 0$ and since $\Omega$ is a Stonean space, and $\mu_{m}$ a normal measure, $\mu_{m}\left(\delta_{\epsilon}^{c}\right)=$ $\mu_{m}\left(\left(\delta_{\epsilon}^{c}\right)^{\circ}\right)$ where $\left(\delta_{\epsilon}^{c}\right)^{\circ}$ is the interior of $\delta_{\epsilon}^{c}$. The same argument as the preceding one shows that there exists an open and closed subset $\sigma_{\epsilon} \subset\left(\delta_{\epsilon}^{c}\right)^{\circ}$ with $\mu_{m}\left(\sigma_{\epsilon}^{c}\right)<\epsilon / 2$. Let $\lambda_{\epsilon}=\rho \cap \sigma_{\epsilon}$. Then, $\mu_{m}\left(\lambda_{\epsilon}\right)<\epsilon$ for all $1 \leq m \leq k$. It follows that all the components of the vectors $l_{i}^{\epsilon}=l_{i} \chi_{\lambda_{\epsilon}} \in L$ are in $E B(\Omega, \Sigma)$. Let $M$ be the matrix whose $i$-th column is $l_{i}^{\epsilon}$. Then, using Lemma 11, it follows that $M$ is a bounded linear operator that commutes with every element in $B$, so $M \in B^{\prime}$. The choice of $\alpha$ implies that $M(w)$ is nonsingular for every $\omega \in \lambda_{\epsilon}$. Consider the matrix $N$ defined as follows:

$$
N(w)=\left\{\begin{array}{cl}
M(w)^{-1} & \text { if } w \in \lambda_{\epsilon}, \\
0 & \text { if } w \in \lambda_{\epsilon}^{c} .
\end{array}\right.
$$

By restricting $N$ to an open and closed subset of $\lambda_{\epsilon}$, if necessary, we can apply Lemma 11 again and get $N \in B^{\prime}$. It follows that the columns of the product $M N$ are linear combinations of vectors in $\mathscr{D}$ with coefficients in $B$. Since $\mathscr{D}$ is invariant under $B$ we have that these columns belong to $\mathscr{D}$. Since $M(w) N(w)=I$ for $w \in \lambda_{\epsilon}$ the proof is completed.

We will use next the following results about spectral operators and their resolutions of the identity from [Dunford and Schwartz 1988]. 
Remark 14. If the operator $M$ commutes with the spectral operator $T$, then $M$ commutes with every resolution of the identity of $T$.

Proof. This is [Dunford and Schwartz 1988, Corollary XV.3.7].

\section{Algebras with complemented invariant subspace lattices}

In this section we will prove our main result:

Theorem 15. Let $B$ be the strongly closed subalgebra of $B(X)$ generated by a complete Boolean algebra of projections $\mathscr{B} \subset B(X)$ of finite uniform multiplicity, $k$, with the direct sum property. If $A \subset B(X)$ is a strongly closed algebra with complemented invariant subspace lattice that contains $B$, then $A$ is reflexive.

The proof of this theorem will be given after a series of auxiliary results. In the rest of this section $\mathscr{B}$ and $B$ will be as in Theorem 15 . We will identify $X$ with $\left(L,\|\cdot\|_{0}\right)$ as in Remark 8.

Proposition 16. Let $B$ as in Theorem 15 and let $T$ be a densely defined closed operator on $X$ which commutes with $B$. There exists an increasing sequence of projections $\left\{q_{p}\right\}_{p=1}^{\infty} \subset \mathscr{B}$ that converges strongly to I such that $T q_{p}$ is a spectral operator of finite type for every $p \in \mathbb{N}$.

Proof. Let $\mathscr{D} \subset X$ be the (dense) domain of $T$. Since $T$ commutes with $B$ it follows that $\mathscr{D}$ is invariant under $B$. By Lemma 13 it follows that for every $p \in \mathbb{N}$ there is an open and closed subset $\sigma_{p} \subset \Omega$ such that $\chi_{\sigma_{p}} \oplus \chi_{\sigma_{p}} \oplus \cdots \oplus \chi_{\sigma_{p}} \in \mathscr{D}$ and $\mu_{l}\left(\sigma_{p}^{c}\right)<1 / 2 p$ for every $1 \leq l \leq k$. Define $r_{p}=S\left(\chi_{\sigma_{p}}\right) \in \mathscr{B}$. Obviously, we can take $r_{p} \leq r_{p+1}$ (in the sense that $r_{p} X \subset r_{p+1} X$ ) for every $p \in \mathbb{N}$. Therefore $\operatorname{Tr}_{p}$ $(p \in \mathbb{N})$ is a bounded operator and $r_{p} \nearrow I$. On the other hand, by Remark 12, since $\operatorname{Tr}_{p} \in B^{\prime}$, for every $p \in \mathbb{N}$, there exists a Borel set $\delta_{p} \in \Sigma$ such that, for all $1 \leq l \leq p$, we have $\mu_{l}\left(\delta_{p}^{c}\right)<1 / 2 p$. Furthermore, if $q_{p}=S\left(\chi_{\delta p \cap \sigma_{p}}\right)$, then $T q_{p}$ is a spectral operator of finite type. Clearly $\left\{q_{p}\right\}$ is an increasing sequence of projections in $\mathscr{B}$ that converges strongly to $I$ and the proof is completed.

Proposition 17. Assume that $B$ is as in the statement of Theorem 15. Let $T$ be a densely defined graph transformation for $B \subset B(X)$. Then there exists an increasing sequence of projections $\left\{q_{p}\right\}_{p=1}^{\infty} \subset \mathscr{B}$ that converges strongly to I such that $T q_{p}$ is a spectral operator of finite type for every $p \in \mathbb{N}$. In particular every such transformation is closable and its closure commutes with $B$.

Proof. Let $T$ be a densely defined graph transformation for $B$ with domain $\mathscr{D}_{T}$. Since $T$ is a graph transformation for $B$, there exists $l \in \mathbb{N}$ and operators $T_{1}, T_{2}, \ldots, T_{l-2}$ such that the subspace

$$
Z=\left\{x \oplus T x \oplus T_{1} x \oplus T_{2} x \oplus \cdots \oplus T_{l-2} x \mid x \in \mathscr{D}_{T}\right\}
$$


belongs to Lat $B^{(l)}$. Define $\Delta_{l-1}=\{x \oplus x \oplus \cdots \oplus \mid x \in X\} \subset X^{(l-1)}$. Then it can be easily seen that the subspace

$$
\Delta_{l-1}^{c}=\left\{x_{1} \oplus x_{2} \oplus \cdots \oplus x_{l-1} \mid x_{i} \in X \text { with } \sum_{i=1}^{l-1} x_{i}=0\right\}
$$

is a Banach subspace complement of $\Delta_{l-1}$ which is invariant under every element of $B^{(l-1)}$. The operator $\widetilde{T}$ defined by

$$
\widetilde{T}(x \oplus x \oplus \cdots \oplus x)=T x \oplus T_{1} x \oplus \cdots \oplus T_{l-2} x \quad \text { if } x \in \mathscr{D}_{T}
$$

and

$$
\widetilde{T}\left(x_{1} \oplus x_{2} \oplus \cdots \oplus x_{l-1}\right)=0 \quad \text { if } x_{1} \oplus x_{2} \oplus \cdots \oplus x_{l-1} \in \Delta_{l-1}^{c}
$$

is a closed, densely defined operator which commutes with $B^{(l-1)}$. An application of Proposition 16 with $k$ replaced by $k(l-1)$ completes the proof.

Remark 18. Let $A \subset B(X)$ be a strongly closed algebra with complemented invariant subspace lattice and $I \in A$. Then, if $Q \in A^{\prime}$ is such that $Q^{2}=0$ it follows that $Q \in(\operatorname{algLat} A)^{\prime}$.

Proof. The proof of [Feintuch and Rosenthal 1973, Lemma 3] for the particular case of Hilbert spaces can be extended to the case of Banach spaces. Indeed, let $Q \in A^{\prime}$ be such that $Q^{2}=0$. Then, if $Y=\operatorname{ker} Q$ is the null space of $Q, Y$ is in Lat $A$ and since $A$ has a complemented invariant subspace lattice, $Y$ has a complement, $Y^{c}$ in Lat $A$. Therefore $Q$ can be written as a matrix

$$
Q=\left[\begin{array}{ll}
0 & c \\
0 & 0
\end{array}\right]
$$

and every $a \in A$ can be written as the matrix

$$
a=\left[\begin{array}{cc}
a_{1} & 0 \\
0 & a_{2}
\end{array}\right] .
$$

Moreover, every $b \in \operatorname{algLat} A$, can be written as a matrix

$$
b=\left[\begin{array}{cc}
b_{1} & 0 \\
0 & b_{2}
\end{array}\right] \text {. }
$$

Since $a Q=Q a$ it follows that $c a_{2}=a_{1} c$. Hence the subspace $\left\{c x \oplus x \mid x \in Y^{c}\right\}$ belongs to Lat $A$ and is therefore invariant for algLat $A$. It follows that $c b_{2}=b_{1} c$, so $Q b=b Q$.

Part (i) of the next result is a generalization of Remark 18.

Proposition 19. Let $A \subset B(X)$ be an algebra with complemented invariant subspace lattice. 
(i) If $Q \in A^{\prime}$ is a nilpotent operator, then $Q \in(\operatorname{algLat} A)^{\prime}$.

(ii) If $T=R+Q$ is a spectral operator of finite type (where $R$ is spectral of scalar type and $Q$ is nilpotent) and $T \in A^{\prime}$, then $R \in(\operatorname{algLat} A)^{\prime}$ and $N \in(\operatorname{algLat} A)^{\prime}$.

Proof. We will prove point (i) of this proposition by induction. By Remark 18, if $Q \in A^{\prime}$ and $Q^{2}=0$, then $Q \in(\operatorname{algLat} A)^{\prime}$. Suppose that for every operator $Q \in A^{\prime}$ with $Q^{n}=0$ it follows that $Q \in(\operatorname{algLat} A)^{\prime}$ and let $Q \in A^{\prime}$ with $Q^{n+1}=0$. Let $p_{0}$ denote a projection on $\operatorname{ker} Q$ such that $p_{0} \in A^{\prime}$. Since $Q p_{0}=0$ it follows that

$$
\left(1-p_{0}\right) Q=\left(1-p_{0}\right) Q\left(1-p_{0}\right)
$$

and therefore

$$
\left(1-p_{0}\right) Q^{k}=\left(\left(1-p_{0}\right) Q\left(1-p_{0}\right)\right)^{k}, \quad k \in \mathbb{N} .
$$

Since $Q^{n+1}=0$ we have $Q^{n}(X) \subset \operatorname{ker} Q$ and therefore

$$
0=\left(1-p_{0}\right) Q^{n}=\left(\left(1-p_{0}\right) Q\left(1-p_{0}\right)\right)^{n} .
$$

By hypothesis, $\left(1-p_{0}\right) Q=\left(1-p_{0}\right) Q\left(1-p_{0}\right) \in(\operatorname{algLat} A)^{\prime}$. On the other hand, since $Q \in A^{\prime}$ and $p_{0} \in A^{\prime}$ we have $p_{0} Q \in A^{\prime}$. Since obviously $\left(p_{0} Q\right)^{2}=0$, by Remark 18, it follows that $p_{0} Q \in(\operatorname{algLat} A)^{\prime}$. Therefore

$$
Q=p_{0} Q+\left(1-p_{0}\right) Q \in(\operatorname{algLat} A)^{\prime}
$$

and the proof of (i) is completed.

We turn now to prove point (ii). By Remark 14, every resolution of the identity of $T, \boldsymbol{E}(\delta)$, where $\delta$ is a Borel subset of the spectrum of $T, \delta \subset \operatorname{sp}(T)$, is in $A^{\prime}$. Therefore, since $A$ has complemented invariant subspace lattice, it follows that $\boldsymbol{E}(\delta) \in(\text { algLat } A)^{\prime}$ for every Borel set $\delta \subset \operatorname{sp}(T)$. Hence $R=\int \lambda \boldsymbol{E}(d \lambda) \in$ (algLat $A)^{\prime}$. Since $T \in A^{\prime}$ and $R \in A^{\prime}$ it follows that $Q \in A^{\prime}$. By part (i) it follows that $Q \in(\operatorname{algLat} A)^{\prime}$.

Lemma 20. Let $A$ be a strongly closed algebra with complemented invariant subspace lattice that contains a complete Boolean algebra of projections of finite uniform multiplicity $k$ with the direct sum property. Then, if $K \in \operatorname{Lat} A^{(n)}$ for some $n \in \mathbb{N}$, then, there exists an increasing sequence of projections $\left\{p_{m}\right\} \subset \mathscr{B}, p_{m} \nearrow I$ such that $p_{m}^{(n)} K$ is complemented in $\operatorname{Lat}\left(p_{m} A p_{m}\right)^{(n)}$ for every $m \in \mathbb{N}$.

Proof. We will prove the lemma by induction on $n$. For $n=1$ the statement is obvious with $p_{m}=I$ for every $m$. Let $K \in$ Lat $A^{(n)}$. Define

$$
K_{0}=\left\{x \in X^{(n-1)} \mid 0 \oplus x \in K\right\} .
$$

Obviously, $K_{0} \in$ Lat $A^{(n-1)}$, so there exists an increasing sequence of projections $\left\{r_{m}\right\} \subset \mathscr{B}, r_{m} \nearrow I$ such that $r_{m}^{(n-1)} K_{0}$ is complemented in $\operatorname{Lat}\left(r_{m} A r_{m}\right)^{(n-1)}$. Let 
$\left(r_{m}^{(n-1)} K_{0}\right)^{c}$ be the complement of $r_{m}^{(n-1)} K_{0}$ in Lat $\left(r_{m} A r_{m}\right)^{(n-1)}$. Then,

$$
\left(r_{m} X \oplus\left(r_{m}^{(n-1)} K_{0}\right)^{c}\right) \cap K \in \text { Lat } A^{(n)}
$$

and

$$
r_{m}^{(n)} K=\left(0 \oplus r_{m}^{(n-1)} K_{0}\right)+\left(r_{m} X \oplus\left(r_{m}^{(n-1)} K_{0}\right)^{c}\right) \cap r_{m}^{(n)} K .
$$

Since $\left(r_{m} X \oplus\left(r_{m}^{(n-1)} K_{0}\right)^{c}\right) \cap r_{m}^{(n)} K$ is the complement of $0 \oplus r_{m}^{(n-1)} K_{0}$ in $r_{m}^{(n)} K$, there exist graph transformations $T_{1}, T_{2}, \ldots, T_{n-1}$ such that

$$
\left(r_{m} X \oplus\left(r_{m}^{(n-1)} K_{0}\right)^{c}\right) \cap r_{m}^{(n)} K=\left\{x \oplus T_{1} x \oplus T_{2} x \oplus \cdots \oplus T_{n-1} x \mid x \in P\right\},
$$

where $P$ is a linear subspace of $r_{m} X$ invariant under every element of $r_{m} A r_{m}$. The closure of $P$ in $r_{m} X, \bar{P}$, belongs to $\operatorname{Lat}\left(r_{m} A r_{m}\right)$ and hence has a complement $\bar{P}^{c}$ in $\operatorname{Lat}\left(r_{m} A r_{m}\right)$. For $1 \leq i \leq n-1$, consider the following densely defined, graph transformation on $r_{m} X$ :

$$
\widetilde{T}_{i} x=\left\{\begin{array}{cl}
T_{i} x & \text { if } x \in P, \\
0 & \text { if } x \in \bar{P}^{c} .
\end{array}\right.
$$

Then $\widetilde{T}_{i}$ commutes with $A$. By Proposition 17 , there exists an increasing sequence of projections $\left\{q_{p}\right\} \subset \mathscr{B}, q_{p} \nearrow I$ such that $\widetilde{T}_{i} q_{p}$ are bounded spectral operators of finite type. From Lemma 3 it follows that the subspace

$$
\left\{q_{p} x \oplus \widetilde{T}_{1} q_{p} x \oplus \widetilde{T}_{2} q_{p} x \oplus \cdots \oplus \widetilde{T}_{n-1} q_{p} x \mid x \in q_{p} P \oplus q_{p} \bar{P}^{c}\right\}
$$

is complemented in $\operatorname{Lat}\left(q_{p} A q_{p}\right)^{(n)}$. By the definition of the transformations $\widetilde{T}_{i}$ it follows immediately that the subspace

$$
\left\{q_{p} x \oplus T_{1} q_{p} x \oplus T_{2} q_{p} x \oplus \cdots \oplus T_{n-1} q_{p} x \mid x \in P\right\}
$$

is complemented in $\operatorname{Lat}\left(q_{p} A q_{p}\right)^{(n)}$. If we set $p_{m}=r_{m} q_{m} \in \mathscr{B}$ we have that $p_{m} \nearrow I$, $p_{m}^{(n)} K$ is complemented in $\operatorname{Lat}\left(p_{m} A p_{m}\right)^{(n)}$ :

$$
\begin{aligned}
p_{m}^{(n)} X=\left(0 \oplus p_{m}^{(n-1)} K_{0}\right)+\left\{p_{m} x \oplus T_{1} p_{m} x \oplus \cdots \oplus T_{n-1} p_{m} x \mid x \in P\right\} & \\
& +\left(\left(p_{m} P\right)^{c} \oplus p_{m}^{(n-1)} K_{0}^{c}\right) .
\end{aligned}
$$

Hence

$$
p_{m}^{(n)} X=\left(p_{m}^{(n)} K\right)+\left(\left(p_{m} P\right)^{c} \oplus p_{m}^{(n-1)} K_{0}^{c}\right),
$$

and the proof of the lemma is completed.

The following statement follows from the proof of Lemma 20.

Remark 21. If $A$ is as in the statement of Lemma 20 and $K \in$ Lat $A^{(n)}$ for some $n \in \mathbb{N}$, then there exists an increasing sequence of projections $\left\{p_{m}\right\} \subset \mathscr{B}, p_{m} \nearrow I$ such that $p_{m}^{(n)} K=\left(0 \oplus p_{m}^{(n-1)} K_{0}\right)+\left\{p_{m} x \oplus T_{1} p_{m} x \oplus \cdots \oplus T_{n-1} p_{m} x \mid x \in P\right\}$, where $K_{0}=\left\{x \in X^{(n-1)} \mid 0 \oplus x \in K\right\}$ and $T_{i} p_{m}, 1 \leq i \leq n-1, m \in \mathbb{N}$, are bounded spectral 
operators of finite type on the closed $A$-invariant subspace $p_{m} P$ that commute with $p_{m} A p_{m}$.

Proof of Theorem 15. Let $b \in \operatorname{algLat} A$ and $K \in$ Lat $A^{(n)}$. We will prove by induction on $n$ that there exists an increasing sequence of projections $\left\{p_{m}\right\} \subset \mathscr{B}$ such that $p_{m} \nearrow I$ and $p_{m}^{(n)} K \in \operatorname{Lat}\left(p_{m} b p_{m}\right)^{(n)}$ for every $m \in \mathbb{N}$ and therefore $K \in$ Lat $b^{(n)}$; then apply Remark 1 to conclude that $b \in A$. By Remark 21, there exists an increasing sequence of projections $\left\{p_{m}\right\} \subset \mathscr{B}, p_{m} \nearrow I$ such that $p_{m}^{(n)} K=$ $\left(0 \oplus p_{m}^{(n-1)} K_{0}\right)+\left\{p_{m} x \oplus T_{1} p_{m} x \oplus T_{2} p_{m} x \oplus \cdots \oplus T_{n-1} p_{m} x \mid x \in P\right\}$ where $K_{0}=$ $\left\{x \in X^{(n-1)} \mid 0 \oplus x \in K\right\}$ and $T_{i} p_{m}, 1 \leq i \leq n-1, m \in \mathbb{N}$, are bounded spectral operators of finite type on the closed $A$-invariant subspace $p_{m} P$ that commute with $p_{m} A p_{m}$. The induction hypothesis and Proposition 2 (i) imply that $0 \oplus p_{m}^{(n-1)} K_{0} \in$ $\operatorname{Lat}\left(p_{m} b p_{m}\right)^{(n)}$. By Proposition 19 (ii) it follows that the bounded spectral operators of finite type $T_{i} p_{m}, 1 \leq i \leq n-1, m \in \mathbb{N}$ commute with $p_{m} b p_{m}$. Hence $p_{m}^{(n)} K \in$ Lat $\left(p_{m} b p_{m}\right)^{(n)}$. Since $p_{m} \nearrow I$ and, by Remark 7 (iv), $\mathscr{B}$ is norm bounded, it follows that $K \in$ Lat $b^{(n)}$ and the result follows.

Corollary 22. Let $A \subset B(X)$ be a strongly closed algebra that contains a complete Boolean algebra of projections $\mathscr{B}$ of finite uniform multiplicity with the direct sum property. If A has complemented invariant subspace lattice, then $A=A^{\prime \prime}$ where $A^{\prime \prime}$ is the bicommutant of $A$.

Proof. Follows from Theorem 15 and Remark 4.

\section{References}

[Arveson 1967] W. B. Arveson, “A density theorem for operator algebras”, Duke Math. J. 34 (1967), 635-647. MR 36 \#4345 Zbl 0183.42403

[Bade 1955] W. G. Bade, "On Boolean algebras of projections and algebras of operators", Trans. Amer. Math. Soc. 80 (1955), 345-360. MR 17,513d Zbl 0066.36202

[Bade 1959] W. G. Bade, "A multiplicity theory for Boolean algebras of projections in Banach spaces”, Trans. Amer. Math. Soc. 92 (1959), 508-530. MR 21 \#7443 Zbl 0086.31501

[Dieudonné 1956] J. Dieudonné, “Champs de vecteurs non localement triviaux”, Arch. Math. (Basel) 7 (1956), 6-10. MR 17,1112c Zbl 0074.10001

[Douglas and Pearcy 1972] R. G. Douglas and C. Pearcy, "Hyperinvariant subspaces and transitive algebras", Michigan Math. J. 19 (1972), 1-12. MR 45 \#4186 Zbl 0233.47005

[Dunford and Schwartz 1988] N. Dunford and J. T. Schwartz, Linear operators, III: Spectral operators, Wiley, New York, 1988. MR 90g:47001c Zbl 0635.47003

[Feintuch and Rosenthal 1973] A. Feintuch and P. Rosenthal, "Remarks on reductive operator algebras", Israel J. Math. 15 (1973), 130-136. MR 48 \#9410 Zbl 0264.46069

[Foguel 1959] S. R. Foguel, "Boolean algebras of projections of finite multiplicity", Pacific J. Math. 9 (1959), 681-693. MR 21 \#7451 Zbl 0087.31701

[Hoover 1973] T. B. Hoover, "Operator algebras with complemented invariant subspace lattices", Indiana Univ. Math. J. 22 (1973), 1029-1035. MR 49 \#1163 Zbl 0259.46054 
[Önder and Orhon 1989] H. Önder and M. Orhon, "Transitive operator algebras on the $n$-fold direct sum of a cyclic Banach space", J. Operator Theory 22:1 (1989), 99-107. MR 90k:47098 Zbl 0701.47024

[Radjavi and Rosenthal 1973] H. Radjavi and P. Rosenthal, Invariant subspaces, Ergebnisse der Math. 77, Springer, New York, 1973. Reprinted Dover, Mineola, 2003. MR 51 \#3924 Zbl 0269.47003

[Rosenthal and Sourour 1977] P. Rosenthal and A. R. Sourour, "On operator algebras containing cyclic Boolean algebras, II”, J. London Math. Soc. (2) 16:3 (1977), 501-506. MR 58 \#17876b Zbl 0391.47028

[Stone 1949] M. H. Stone, "Boundedness properties in function-lattices", Canadian J. Math. 1 (1949), 176-186. MR 10,546a Zbl 0032.16901

[Tzafriri 1967] L. Tzafriri, “Operators commuting with Boolean algebras of projections of finite multiplicity”, Pacific J. Math. 20 (1967), 571-587. MR 34 \#8197 Zbl 0147.12004

Received April 3, 2012.

FLORENCE MERLEVÈDE

UPEM, LAMA, UMR 8050 CNRS

UNIVERSITÉ PARIS EST

BÂTIMENT COPERNIC

5 BouleVard Descartes

77435 CHAMPS-SUR-MARNE

FRANCE

florence.merlevede@u-pem.fr

Costel Peligrad

Department of MATHEMATICAL SCIENCES

UNIVERSITY OF CINCINNATI

PO BOX 210025

Cincinnati, OH 45221-0025

UNITED STATES

peligrc@ucmail.uc.edu

Magda Peligrad

DEPARTMENT OF MATHEMATICAL SCIENCES

UNIVERSITY OF CINCINNATI

PO BOX 210025

CinCINNATI, OH 45221-0025

UNITED STATES

peligrm@ucmail.uc.edu 


\title{
PACIFIC JOURNAL OF MATHEMATICS
}

\author{
msp.org/pjm
}

Founded in 1951 by E. F. Beckenbach (1906-1982) and F. Wolf (1904-1989)

\section{EDITORS}

V. S. Varadarajan (Managing Editor)

Department of Mathematics

University of California

Los Angeles, CA 90095-1555

pacific@math.ucla.edu

Paul Balmer

Department of Mathematics

University of California

Los Angeles, CA 90095-1555

balmer@math.ucla.edu

Daryl Cooper

Department of Mathematics

University of California

Santa Barbara, CA 93106-3080 cooper@math.ucsb.edu

Jiang-Hua $\mathrm{Lu}$

Department of Mathematics

Pokfulam Rd., Hong Kong jhlu@maths.hku.hk
The University of Hong Kong

Don Blasius

Department of Mathematics University of California

Los Angeles, CA 90095-1555

blasius@math.ucla.edu

Robert Finn

Department of Mathematics Stanford University

Stanford, CA 94305-2125

finn@math.stanford.edu

Sorin Popa

Department of Mathematics

University of California

Los Angeles, CA 90095-1555 popa@math.ucla.edu

Paul Yang

Department of Mathematics Princeton University

Princeton NJ 08544-1000

yang@math.princeton.edu

\section{PRODUCTION}

Silvio Levy, Scientific Editor, production@msp.org

\section{SUPPORTING INSTITUTIONS}

ACADEMIA SINICA, TAIPEI

CALIFORNIA INST. OF TECHNOLOGY

INST. DE MATEMÁTICA PURA E APLICADA

KEIO UNIVERSITY

MATH. SCIENCES RESEARCH INSTITUTE

NEW MEXICO STATE UNIV.

OREGON STATE UNIV.

\author{
STANFORD UNIVERSITY \\ UNIV. OF BRITISH COLUMBIA \\ UNIV. OF CALIFORNIA, BERKELEY \\ UNIV. OF CALIFORNIA, DAVIS \\ UNIV. OF CALIFORNIA, LOS ANGELES \\ UNIV. OF CALIFORNIA, RIVERSIDE \\ UNIV. OF CALIFORNIA, SAN DIEGO \\ UNIV. OF CALIF., SANTA BARBARA
}

\author{
Vyjayanthi Chari \\ Department of Mathematics \\ University of California \\ Riverside, CA 92521-0135 \\ chari@math.ucr.edu \\ Kefeng Liu \\ Department of Mathematics \\ University of California \\ Los Angeles, CA 90095-1555 \\ liu@math.ucla.edu \\ Jie Qing \\ Department of Mathematics \\ University of California \\ Santa Cruz, CA 95064 \\ qing@cats.ucsc.edu
}

These supporting institutions contribute to the cost of publication of this Journal, but they are not owners or publishers and have no responsibility for its contents or policies.

See inside back cover or msp.org/pjm for submission instructions.

The subscription price for 2014 is US \$410/year for the electronic version, and \$535/year for print and electronic.

Subscriptions, requests for back issues and changes of subscribers address should be sent to Pacific Journal of Mathematics, P.O. Box 4163, Berkeley, CA 94704-0163, U.S.A. The Pacific Journal of Mathematics is indexed by Mathematical Reviews, Zentralblatt MATH, PASCAL CNRS Index, Referativnyi Zhurnal, Current Mathematical Publications and Web of Knowledge (Science Citation Index).

The Pacific Journal of Mathematics (ISSN 0030-8730) at the University of California, c/o Department of Mathematics, 798 Evans Hall \#3840, Berkeley, CA 94720-3840, is published twelve times a year. Periodical rate postage paid at Berkeley, CA 94704, and additional mailing offices. POSTMASTER: send address changes to Pacific Journal of Mathematics, P.O. Box 4163, Berkeley, CA 94704-0163.

PJM peer review and production are managed by EditFLOW ${ }^{\circledR}$ from Mathematical Sciences Publishers.

\section{PUBLISHED BY}

mathematical sciences publishers

nonprofit scientific publishing

http://msp.org/

(C) 2014 Mathematical Sciences Publishers 


\section{PACIFIC JOURNAL OF MATHEMATICS}

Volume $267 \quad$ No. $2 \quad$ February 2014

Sums of squares in algebraic function fields over a complete discretely valued field

KARIM JOHANNES BECHER, DAVID GRIMM and JAN VAN GEEL

On the equivalence problem for toric contact structures on $\mathbf{S}^{\mathbf{3}}$-bundles over $S^{2}$

\section{Charles P. Boyer and Justin PATi}

An almost-Schur type lemma for symmetric $(2,0)$ tensors and applications

Xu CHENG

Algebraic invariants, mutation, and commensurability of link complements

ERIC CHESEBRO and JASON DEBLOIS

Taut foliations and the action of the fundamental group on leaf spaces and universal circles

YOSUKE KANO

A new monotone quantity along the inverse mean curvature flow in $\mathbb{R}^{n}$

KwoK-Kun Kwong and Pengzi Miao

Nonfibered L-space knots

TYE LIDMAN and LIAM WATSON

Families and Springer's correspondence

GEORGE LUSZTIG

Reflexive operator algebras on Banach spaces

Florence Merlevède, Costel Peligrad and Magda Peligrad Harer stability and orbifold cohomology

NiCOLA PAGANI

Spectra of product graphs and permanents of matrices over finite rings

LE ANH VINH

The concavity of the Gaussian curvature of the convex level sets of minimal surfaces with respect to the height

PEI-HE WANG 